\title{
5G Traffic Prediction with Time Series Analysis
}

\author{
Nikhil Nayak, Rujula Singh R
}

\begin{abstract}
In today's day and age, a mobile phone has become a basic requirement needed for anyone to thrive. With the cellular traffic demand increasing so dramatically, it is now necessary to accurately predict the user traffic in cellular networks, to improve the performance in terms of resource allocation and utilization. Since traffic learning and prediction is a classical and appealing field, which still yields many meaningful results, there has been an increasing interest in leveraging Machine Learning tools to analyze the total traffic served in each region, to optimize the operation of the network. With the help of this project, we seek to exploit the traffic history by using it to predict the nature and occurrence of future traffic. Furthermore, we classify the traffic into application types, to increase our understanding of the nature of the traffic. By leveraging the power of machine learning and identifying its usefulness in the field of cellular networks we try to achieve three main objectives - classification of the application generating the traffic, prediction of packet arrival intensity and burst occurrence. The design of the prediction and classification system is done using Long Short Term Memory (LSTM) model. The LSTM predictor developed in this experiment would return the number of uplink packets and estimate the probability of burst occurrence in the specified future time interval. For the purpose of classification, the regression layer in our LSTM prediction model is replaced by a SoftMax classifier which is used to classify the application generating the cellular traffic into one of the four applications including surfing, video calling, voice calling, and video streaming.

Keywords: Classification, K-Means, Time series analysis, Traffic prediction.
\end{abstract}

\section{INTRODUCTION}

I the current era, mobile phones have become a basic requirement needed for everyone to carry, on a day-to-day basis. With the cellular traffic demand increasing so dramatically, it is now necessary to accurately predict the user traffic in cellular networks, to improve the performance in terms of resource allocation and resource utilization. Since traffic learning and prediction is a classical and appealing field, which still yields many meaningful results, there has been an increasing interest in leveraging Machine Learning to analyze the total traffic served in a given region, to optimize the operation of the network. Based on the analysis, we can exploit the traffic history by using it to predict the nature and occurrence of future traffic.

Revised Manuscript received on October 17, 2021.

Manuscript published on October 30, 2021.

Nikhil Nayak*, Department of Computer Science \& Engineering, RV College of Engineering. Bengaluru, (Karnataka), India

Rujula Singh R, Department of Computer Science \& Engineering, RV College of Engineering. Bengaluru, (Karnataka), India

(c) The Authors. Published by Blue Eyes Intelligence Engineering and Sciences Publication (BEIESP). This is an open access article under the CC BY-NC-ND license (http://creativecommons.org/licenses/by-nc-nd/4.0/)
Manuscript received on October 11, 2021.

* Correspondence Author

Furthermore, we classify the traffic into application types, to increase our understanding of the nature of the traffic.

\section{OBJECTIVE}

With the help of Machine Learning, we can identity various trends and patterns in $5 \mathrm{G}$ cellular networks. In this project we strive to achieve four main objectives as follows:

- Prediction of burst occurrence, i.e., prediction of whether there will be a sudden spike in traffic flow at a given moment of time.

- Prediction of the intensity of packet arrival at a given instant of time.

- Analysis of inter-arrival time for all packets within a total period of 7 days.

- Categorize the packet flow into 4 parts, using a clustering algorithm.

\section{RELATED WORK}

In [2], the dynamics of cellular traffic along with the nuances for predicting future demands to improve resource efficiency are understood. Various methods of time series forecasting for cellular traffic using Convolutional and Recurrent Neural Networks are explored in [6]. In [8], the spatial and temporal correlations of the cellular traffic in different time periods and neighboring cells, respectively, have been explored using neural networks in order to improve the accuracy of traffic prediction. The authors of [7] propose a method to combine CNNs and RNNs to extract more information and yield better results in the field of time series forecasting for cellular traffic. In [4, 9], solutions for time series analysis using LSTMs are presented, but the analysis is weak, and they are lacking in technical details and do not mention the set of features that have been used for the training and analysis. Various other papers [12, 10, 11, 4, 9, 5] have been used for technical details and ideas, helping us with the work we have done as a part of this project. The paper [3] serves as our base and we use it to model our implementation.

\section{PRELIMINARIES}

\section{A. 5G Data}

5G is the fifth-generation technology standard for broadband cellular networks. It is designed to increase speed, reduce latency, and improve flexibility of wireless services.

$5 \mathrm{G}$ is a unified, more capable air interface. It has been designed with an extended capacity to enable next-generation user experiences, empower new deployment models and deliver new services. Its goal is to connect virtually everyone and everything together including machines, objects, and devices.

Published By:

Blue Eyes Intelligence Engineering and Sciences Publication (BEIESP)

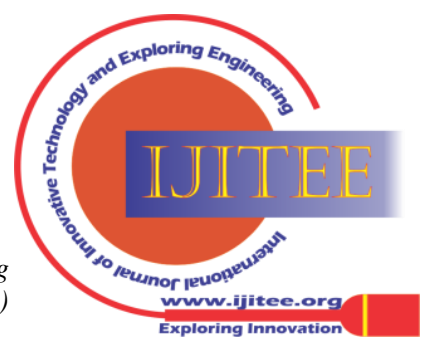




\section{B. Time Series Analysis}

A time series is simply a series of data points ordered in time. In a time series, time is often the independent variable and the goal is usually to make a forecast for the future. Time Series analysis can be useful to see how a given asset, security or economic variable changes over time. Using characteristics of time series like autocorrelation, stationarity and seasonality, predictions for future time intervals are made based on the data at previous instances of time. Meaningful statistics and characteristics of data can also be brought out using time series analysis.

\section{Time Series Analysis For Cellular Traffic}

Time Series Analysis can be used to detect malicious data in cellular traffic and detect any anomalies. It can be used to predict and model the behavior of cellular networks, so as to understand the traffic and cause of traffic. This technique is furthermore used for dynamic resource allocation by predicting unusual network load and then using other models to reallocate traffic accordingly. Time Series analysis is best suited to predict occurrence of bursty traffic and to estimate the intensity of the burstiness.

\section{Long Short-Term Memory}

Long Short-Term Memory (LSTM) networks are a type of recurrent neural network capable of learning order dependence in sequence prediction problems. LSTMs are capable of learning and using long term dependencies. LSTM units have a cell, an input gate, an output gate and a forget gate. The cells remember information over arbitrary time intervals and the three gates regulate the flow of information into an out of the cell. They can be used for text analysis, speech recognition, language modulation, time series analysis and many other applications.

\section{E. LSTM - Time Series Analysis}

LSTMs can be used for time series analysis where they look at time series data and learn to make predictions from them. The LSTMs take in multiple rows of data as a subset and then learn from that in every step. These variants of RNNs have proven to be better learners and capture time related trends much better than most of its counterparts.

\section{METHODOLOGY}

In this section, we focus on the explanation of various background requirements, the proposed approach and the implementation of the final model.

\section{A. Data Preprocessing Cleaning}

The dataset has been taken from [1]. Here, raw data was generated using a variety of applications and captured using Wire Shark. This data was stored in MATLAB Class objects and had to be extracted using MATLAB. The extracted data had to be converted to the right format for columns like Timestamp and Na N values had to be dropped. The source and destination IP Addresses were analyzed to identify the user addresses and the tower address. Based on the IP addresses and direction of flow, the packets were labelled as Uplink and Downlink Packets. The packets are grouped together based on the timestamp to incorporate all the packets within a given period, for example, 1s. The total number of uplink and downlink packets are aggregated for every time

group and the length of packets are also considered for further processing. The total amount of data transmitted in that given time period (ex. 1s) is then calculated. The final features are Uplink/Downlink, Count, Size of Uplink/Downlink, Uplink Downlink Ratio, Protocol and the total amount of data transmitted serves as the target variable.

\section{B. Network Architecture}

We use a Deep Learning model to predict whether the traffic will have sudden spikes in intensity and how severe these particular spikes are. For this, we need a thorough network of neurons. We employ the following as a part of our model.

- $\quad$ The output layer is a Regression layer as we need to predict real values.

- An LSTM layer comprising of 100 units/elements.

- The LSTM layer is followed by a Fully Connected layer.

\section{PROPOSED APPROACH}

- The cellular traffic dataset contains the incoming/outgoing packets captured at the Internet protocol (IP) level with help of packet capture tool e.g. WireShark.

- Prediction of the burst occurrence is done using Long Short Term Memory (LSTM) model.

- Root Mean Square Error (RMSE) and a mapping of the actual values to the predicted values are chosen as the performance indicators for the prediction of traffic intensity.

- Accuracy, recall, F1 Score and precision are the performance indicators for the prediction of the occurrence of a burst.

- The LSTM network returns the number of uplink packets and estimates the probability of burst occurrence in a specified time interval. Occurrence of a burst is predicted by setting a burst threshold.

A brief overview of the entire process can be seen in Fig.1. It is a Flowchart which brings out the significance of various steps that we have taken in this project.

\section{IMPLEMENTATION}

\section{A. Preprocessing Implementation}

- FeatureExtraction: This is to convert the raw data into meaningful data using Feature Extraction techniques.

- ZeroPadding: The is used to pad the data with 0 rows, so as to make the data meaningful without any inconsistencies.

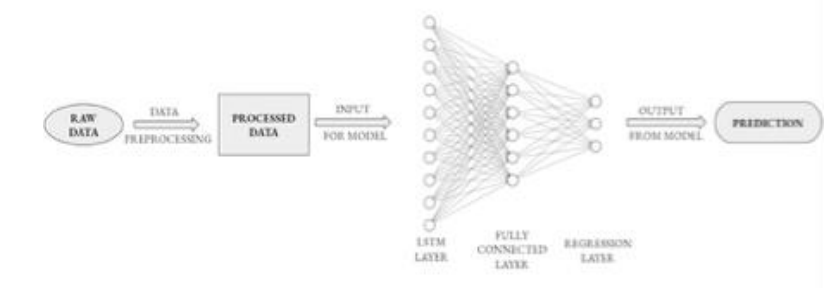

Fig. 1. A Flowchart Depicting Our Proposed Approach

Published By:

Blue Eyes Intelligence Engineering and Sciences Publication (BEIESP)

(C) Copyright: All rights reserved.

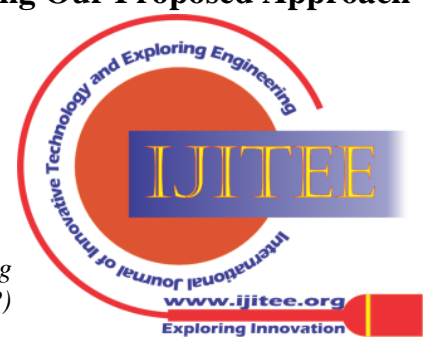


- ProPadding: The is used to pad zeroes in a special way, so as to reduce the bias towards 0s and prevent the meaningful data from being treated like outliers.

\section{B. Implementation of LSTM}

- LSTM Implementation 1x60: This is used to train and evaluate the LSTM on data with bin size of 1s and history of 60 rows.

- LSTM Implementation 3x20: This is used to train and evaluate the LSTM on data with bin size of 3s and history of 20 rows.

- LSTM Implementation 60x1: This is used to train and evaluate the LSTM on data with bin size of 60s and history of a single row.

- Pro Padding Implementation: This is used to train the LSTM on data padded with limited 0s to reduce bias, with bin size of 1 s and history of 10 rows.

\section{Implementation of LSTM Clustering}

- LSTM Implementation NonZeroPrediction Burstiness: This is used to predict only nonzero values, so as to capture the trend of the data and not get influenced by the large number of zeroes, using bin size of 1s and history of 10s.

- KMeans Clustering Cellular Traffic Variation: This is used to compare the results of KMeans clustering with and without Uplink Downlink Ratio for bin sizes of 1s, 30s and 60s with a history of a single row.

\section{RESULTS AND DISCUSSION}

\section{A. Evaluation Metrics}

To test the goodness of the model, two evaluation metrics were used. The evaluation metrics encompass both the mathematical aspects as well as visual aspects: Root Mean Squared Error (RMSE), it is the standard deviation of the residuals. It tells you how concentrated the data is around the line of best fit. Root mean square error is commonly used in climatology, forecasting, and regression analysis to verify experimental results. Graph, the graph comparing the predicted values for Uplink Count with the Actual values of the Uplink Count is a very good way to evaluate whether or not the predictions are accurate and are fitting the general trend line. RMSE does not prove to be good metric by itself. While predicting values, if the model is biased to zeroes and flatlines by predicting only zero values, then the error might not be very large, as there are very few peaks and do not contribute to errors significantly. On the other hand, even if the RMSE is very high, we cannot say that the model has failed, because the large error might be the cumulative result of extremely small and acceptable deviations from the zero value, for every single zero value. We try to gauge our model with the help of a graph, to see the goodness of fit and then decide the next step of action. Even the graphical method cannot be solely sufficient as we cannot estimate the true error by looking at just the graph. Hence, we use the coupled combination of the RMSE score and the graph, to get a true understanding of the model and its behavior.

\section{B. Results of LSTMs}

- LSTM Implementation 1x60: The model gave a very good error score but the graph was a flat line and the model had learnt to predict only zeroes, as a result of which, the RMSE was quite low. This shows that the model was being highly influenced by the zero padded rows and had developed bias.

- LSTM Implementation 3x20: This model also faced the same issue as the above model, where it developed a bias towards the zeroes and predicted a flat line with low error score. This led to the understanding that a larger bin size might be helpful.

- LSTM Implementation 60x1: The model successfully learnt the trend line but could not predict bursts and could not reach the peaks as it was still being trained on too many zeroes. The error was quite high as it was not predicting zeroes for the zero values and was not reaching peaks as well. It was stuck in the middle. This showed the total 60s interval was not a suitable parameter.

- Pro Padding Implementation: To eliminate the bias introduced by the zero padded rows, the padding was done in a special way. The model gave good results for RMSE but the trend line was still flat, as the bin size was 1s and the model was learning to predict zeroes as most bins were empty due to the small size of bins.

\section{Results of LSTM and K-Means}

- LSTM Implementation NonZeroPrediction Burstiness: Since the previous models were being trained on zero values, we adjusted the training set so that the model learns from the zero padded history but does not get influenced by trying to predict the zeroes. The model gave excellent results as the RMSE had been significantly lowered and even the trend line was matched almost completely. The burstiness was also predicted by applying a threshold to the predicted value. The threshold was taken as the mean of all the Uplink packet counts in the training set. The accuracy of the prediction of burst was also measured and this gave us excellent results.

This shows that our model was successfully predicting bursts, as the classification metrics suggested, and was able to predict the intensity of the traffic as well, as is indicated by the error score and the graph. The confusion matrix can be calculated using the values of the parameters in the classification report. The results of the model can be seen in Fig.2.

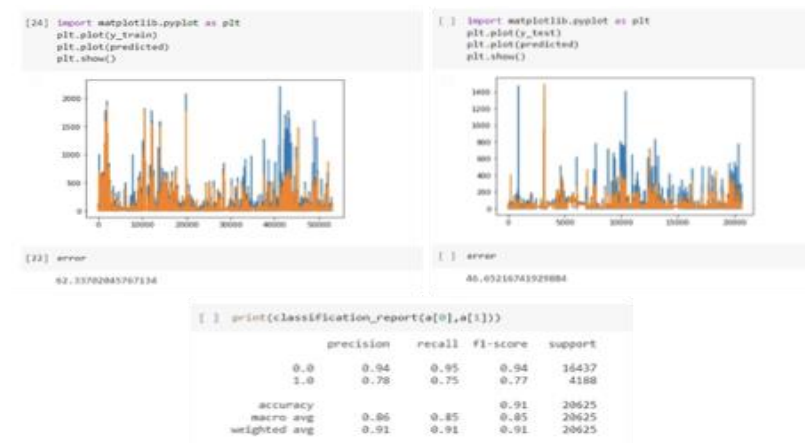

Fig. 2. The RMSE and the Graph of the trend line for the Training and Testing sets respectively, followed by the Classification Report, quantifying the quality of the burst prediction.

Published By:

Blue Eyes Intelligence Engineering and Sciences Publication (BEIESP)

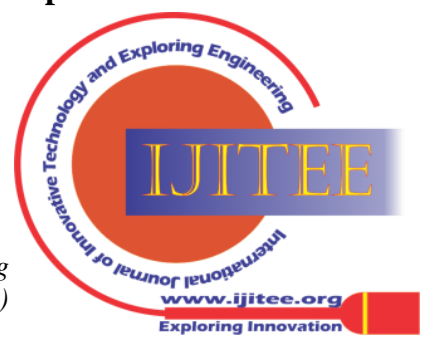


- KMeans CLutering Cellular Traffic Variation: Clustering was performed on the entire dataset for various time bins employing various feature sets. The zero padded values were not included as they do not provide any value to the clustering algorithm. Time bins of $1 \mathrm{~s}, 30$ s and 60 s were used, each with two different subsets of features. The first subset comprises of the 'UplinkCount' and the 'DownlinkCount'. The second subset adds an additional feature called the 'Uplink/DownlinkRatio'. The general trend of clustering is similar across all the variations. Although the clustering could not be validated because of the lack of target variables, it is quite clear that the clustering was successful as the clusters in all the cases were quite similar and followed the same general trend. Fig. 3 focuses on clustering the data when it was divided into time bins of 1s. Fig. 4 is used to portray the results of clustering obtained when the data was compacted into time bins of 30s and finally Fig.5 shows the results of clustering when the data was aggregated into bin sizes of a minute.

\section{CONCLUSION}

The prediction of traffic burst occurrence and intensity for cellular networks has been investigated, which leverages various machine learning tools. A comprehensive comparative analysis using prediction tools, such as LSTMs, has been carried out, under different traffic circumstances and design parameter selections. The practicality of the developed LSTM model for prediction of burstiness has also been investigated. Our project dives deep into various parameters to understand which model is the most suitable. Reasons for the success or failure of all the models have been understood and presented as a part of this project. Furthermore, data has been clustered into 4 applications, satisfactory and consistent with our theoretical observations.
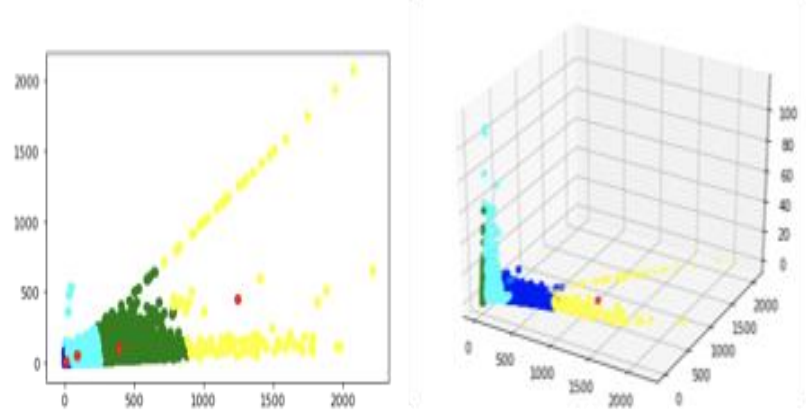

Fig. 3 . The clusters and their centers for a time bin of 1 second.
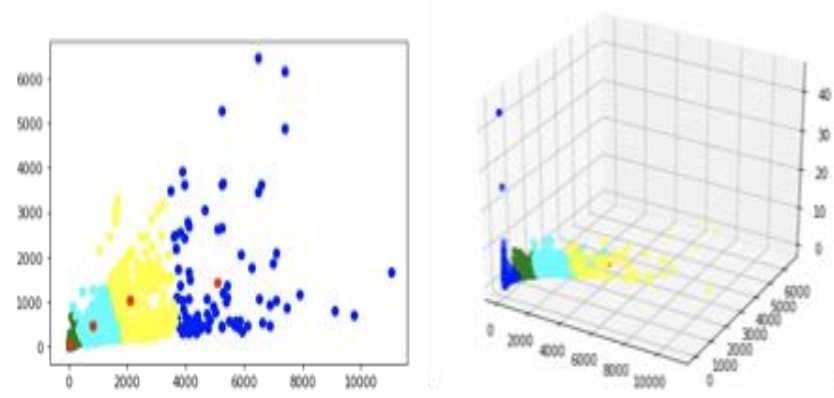

Fig. 4. The clusters and their centers for a time bin of 30 seconds based on how the data was generated. The results are quite

\section{FUTURE WORK}

In the future, we would like to generate our own data as it would be more consistent and complete. We would also have the necessary information regarding the class of applications generating this data. This would help us in achieving better overall quantitative results, which includes, but is not limited to higher accuracy prediction for bigger peaks. Furthermore, supervised classification of the data, into the categories of applications generating the same can be done using our generated data. Future prospects for this project can also include analysis of $5 \mathrm{G}$ data from various IOT devices, which we have currently omitted for the purpose of simplicity. Time Series Analysis oriented towards IOT can be carried out and feasible solutions to the various inherent problems present in the same field can be found.
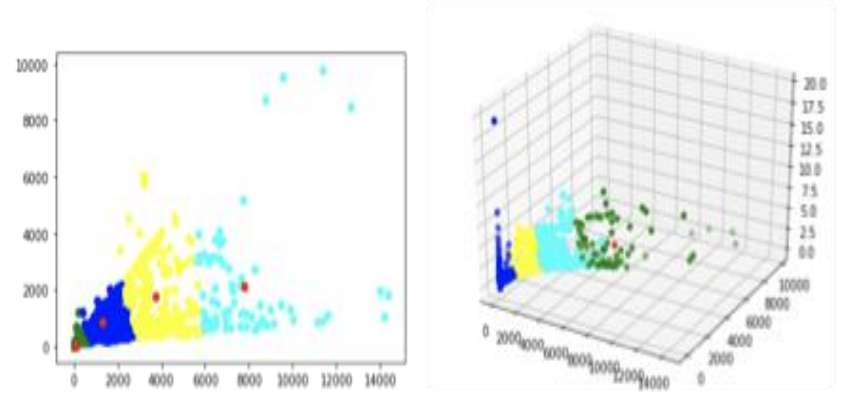

Fig. 5. The clusters and their centres for a time bin of 60 seconds.

\section{REFERENCES}

1. Amin Azari, Mustafa Ozger, and Cicek Cavdar. "Risk-aware resource allocation for URLLC: Challenges and strategies with machine learning”. In: IEEE Communications Magazine 57.3 (2019), pp. 42-48.

2. Amin Azari et al. "Cellular Traffic Prediction and Classification: a comparative evaluation of LSTM and ARIMA". In: International Conference on Discovery Science. Springer. 2019, pp. 129-144.

3. Abdelhadi Azzouni and Guy Pujolle. "NeuTM: A neural network-based framework for traffic matrix prediction in SDN". In: NOMS 2018-2018 IEEE/IFIP Network Operations and Management Symposium. IEEE. 2018, pp. 1-5.

4. Chih-Wei Huang, Chiu-Ti Chiang, and Qiuhui Li. "A study of deep learning networks on mobile traffic forecasting". In: 2017 IEEE 28th Annual International Symposium on Personal, Indoor, and Mobile Radio Communications (PIMRC). IEEE. 2017, pp. 1-6.

5. Guokun Lai et al. "Modeling long-and short-term temporal patterns with deep neural networks". In: The 41st International ACM SIGIR Conference on Research Development in Information Retrieval. 2018, pp. 95-104.

6. Chen Qiu et al. "Spatio-temporal wireless traffic prediction with recurrent neural network". In: IEEE Wireless Communications Letters 7.4 (2018), pp. 554-557.

7. Hoang Duy Trinh, Lorenza Giupponi, and Paolo Dini. "Mobile traffic prediction from raw data using LSTM networks". In: 2018 IEEE 29th Annual International Symposium on Personal, Indoor and Mobile Radio Communications (PIMRC). IEEE. 2018, pp. 1827-1832.

8. Jing Wang et al. "Spatiotemporal modeling and prediction in cellular networks: A big data enabled deep learning approach". In: IEEE INFOCOM 2017-IEEE Conference on Computer Communications. IEEE. 2017, pp. 1-9.

9. $\mathrm{Xu}$ Wang et al. "Spatio-temporal analysis and prediction of cellular traffic in metropolis". In: IEEE Transactions on Mobile Computing 18.9 (2018), pp. 2190-2202.

10. Ying Zhang and Ake Arvidsson. "Understanding the characteristics of cellular data traffic". In: Proceedings of the 2012 ACM SIGCOMM workshop on Cellular networks: operations, challenges, and future design. 2012, pp. 13-18.

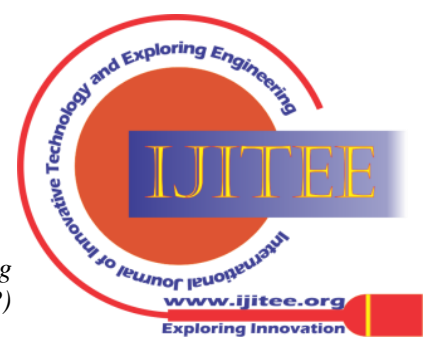




\section{AUTHORS PROFILE}

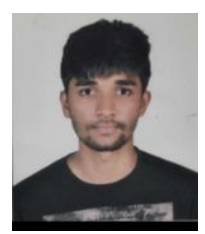

Nikhil Nayak, $\mathrm{He}$ is a Computer Science \& Engineering student at RV College of Engineering. He is a Machine Learning and Artificial Intelligence enthusiast. He is highly motivated and determined to build projects to solve real world problems. He has multiple publications and projects in the field of Machine Learning.

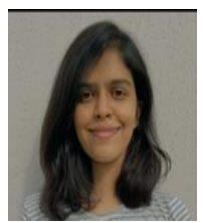

Rujula Singh $\mathbf{R}$, She is a Computer Science \& Engineering student at RV College of Engineering. She is a Machine Learning and Artificial Intelligence enthusiast. She is highly motivated and determined to build projects to solve real world problems. She has multiple publications and projects in the field of Machine Learning.

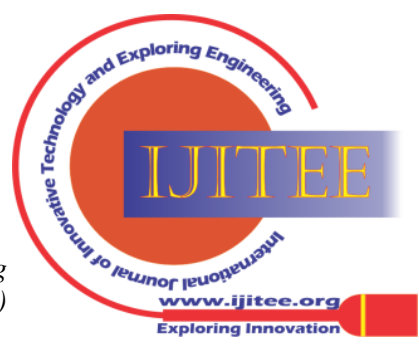

